BI-TP 2012/33

\title{
Taste symmetry breaking at finite temperature
}

\author{
Edwin Laermann and Fabrizio Pucci \\ Fakultät für Physik, Universität Bielefeld, D-33615 Bielefeld, Germany
}

February 14, 2018

\begin{abstract}
The breaking of the taste symmetry is studied in the temperature range between $140 \mathrm{MeV}$ to $550 \mathrm{MeV}$. In order to investigate this violation we have calculated the screening masses of the various taste states fitting the exponential decay of the spatial correlators. The computation has been performed using dynamical $N_{f}=2+1$ gauge field configurations generated with the p4 staggered action along the Line of Constant Physics (LCP) defined by a pion mass $m_{\pi}$ of approximately $220 \mathrm{MeV}$ and the kaon mass $m_{K}$ equals $500 \mathrm{MeV}$. For temperatures below the transition an agreement with the predictions of the staggered chiral perturbation theory has been found and no temperature effect can be observed on the taste violation. Above the transition the taste splitting still shows an $\mathcal{O}\left(a^{2}\right)$ behavior but with a temperature dependent slope. In addition to the analysis done for the pion multiplet we have performed an analogous computation for the light-strange and strange mesons and also looked at the scalar, vector and axial vector channels to understand how the multiplets split at finite temperature. Finally the temperature dependence of the pion decay constant $f_{\pi}$ is investigated to get further information about the chiral symmetry restoration.
\end{abstract}

PACS. 11.15.Ha , 11.10.Wx, 12.38.Gc, 12.38.Mh, 12.39.Fe

\section{Introduction}

One of the most famous problems related to the lattice discretization of quantum chromodynamics (QCD) is the presence of unphysical fermionic modes called doublers. Several methods have been proposed in the literature to avoid this proliferation and to reduce their number. One of these proposals is the well known and widely used staggered fermion formulation introduced for the first time in 11 by Kogut and Susskind. Indeed after the staggering, the sixteen doublers reduce to four species that are usually called tastes to distinguish them from the ordinary flavors.

This construction has become popular in simulations of lattice QCD, in particular at finite temperature because it is computationally cheaper and preserves a remnant of the chiral symmetry. However, since the doubling problem is not completely solved, to reduce further the tastes and restore the correct number of degrees of freedom a rooting procedure has to be introduced. The validity of this technique has long been debated in the literature [2, 3, 4, 5, 6, 7, 8, 9, 10, 11, 12, 13, and even if a definite conclusion is still missing, numerical evidence seems to confirm its correctness namely that the non-local terms appearing at finite lattice spacing become irrelevant in the continuum limit. At non-zero lattice spacing, due to the fact that for staggered fermions the spin and the taste degrees of freedom are mixed, a breaking of the continuum taste symmetry occurs and as a consequence only one of the sixteen pi- ons is a true Goldstone boson. The other fifteen states split according to their transformation properties under the symmetry group preserved by the staggered formulation [14,15,16. Since these taste violations contribute to the cut-off dependence of physical quantities computed on the lattice, several ways have been introduced and applied in the literature to reduce them and to improve the staggered action. In fact, in the p4, aqstad, stout and HISQ actions this improvement was obtained by using some variants of the gauge-link smearing [18, 19, 20, 21, 22 .

In order to have a better comprehension of the impact of these cut-off effects on physical observables it is important to understand how taste symmetry is violated at finite lattice spacing. The concrete way to do that is usually to look at the so called taste splitting defined by the difference between the square of the mass of a non-Goldstone pion and the Goldstone one $\Delta_{\xi}=m_{[\xi]}^{2}-m_{\xi_{5}}^{2}[$. At zero temperature the situation is quite clear. All the results, see e.g. [15, 16, 17] for the most recent investigations, confirm that in the pion sector the taste splitting goes like $a^{2}$ where $a$ is the lattice spacing. Remarkably this behavior can be well described with a tree-level computation in staggered chiral perturbation theory $(\mathrm{S} \chi \mathrm{PT})$ [23, 24, 25]. Indeed in the staggered chiral Lagrangian taste-breaking four-fermion operators appear, physically due to the fact that the exchange of gluons with momenta $q \sim \pi / a$ can

\footnotetext{
${ }^{1}$ In the spinor-taste basis we will indicate with $\gamma$ and $\xi$ the spin and the taste gamma matrices respectively.
} 
change the taste of the staggered quarks.

At finite temperature the situation is not so well understood. $\mathrm{S} \chi \mathrm{PT}$ is formulated at zero temperature and can not help in the understanding of taste symmetry breaking because in principle the structure of the chiral Lagrangian could be modified at finite $T<T_{c}$, where $T_{c}$ is the temperature of approximate restoration of chiral symmetry, possibly leading to temperature effects. For temperatures above the transition we expect the breakdown of $\mathrm{S} \chi \mathrm{PT}$. The aim of this investigation is thus to go further into the problem and to study the dependence of taste breaking on lattice spacing as well as temperature. In order to extract this information we will calculate the screening masses of the different taste components of the pion multiplet at finite temperature in $2+1$ flavor QCD using an improved $\mathrm{p} 4$ [18] staggered fermion action at bare quark masses which correspond to a Goldstone pion mass of $220 \mathrm{MeV}$ and a kaon mass of $500 \mathrm{MeV}$ at zero temperature. The temperature will range from about $140 \mathrm{MeV}$ to $550 \mathrm{MeV}$ and our study will be performed at two different sets of the lattice spacing corresponding to $N_{\tau}=6$ and 8 . Our analysis will not be restricted to the pion sector, we have studied also the scalar, the axial-vector and the vector channels to obtain useful indications about taste violations also in those channels. Additionally we address the effect of the flavors on the taste breaking, analyzing some local and non-local pseudoscalar operators in light-strange $(\bar{u} s)$ and strangestrange $(\bar{s} s)$ channels.

The screening mass analysis is relevant not only for the understanding of the taste symmetry breaking but also for the study of the chiral symmetry. It is a well known fact that an advantage of the staggered formulation is to preserve a $U(1)_{\epsilon}$ subgroup 2 of the chiral symmetry in the continuum. Since this subgroup relates different classes of mesonic operators, in the symmetric phase it predicts the degeneracy between certain channels. Above the transition its restoration demands that at zero quark mass

$$
C(z)_{A V}=(-1)^{z} C(z)_{V}
$$

where $C_{V}$ and $C_{A V}$ are the correlators in the vector $\left(\gamma_{\mu}\right)$ and axial vector $\left(\gamma_{5} \gamma_{\mu}\right)$ channels respectively.

Finally there is a general motivation to study screening masses since from them one can extract information about some non-perturbative properties of the quark - gluon plasma. Indeed the inverse of the screening mass describes the distance beyond which the effect of a test hadron put into the medium is screened.

The plan of the paper is the following: after an introduction to staggered mesons (section 2) where we briefly discuss the symmetry properties of the staggered formulation both at zero and finite temperature, we present a summary of the predictions of staggered chiral perturbation theory on the taste breaking (section 3). Section (4) is devoted to the presentation of the results regarding the screening masses of the different mesonic states at finite temperature before in (5) we will summarize our results, discuss

\footnotetext{
${ }^{2}$ This subgroup should not be confused with the anomalous
} $U(1)_{A}$ of continuum QCD. some open problems and suggest possible directions for a future investigation.

\section{Staggered Mesons}

The symmetry properties of the system are fundamental to the analysis of the correlation functions and the determination of the screening masses. In fact all the states can be classified according to their transformation properties under the subgroup preserved by the lattice discretized theory. For these reasons in this section we review some basic and known facts about the classification of the staggered meson correlators starting with zero temperature [14,28] and then passing to the finite temperature case 29 .

In the continuum limit the symmetry group of one staggered fermion is $S U(2) \times U(4)$ with the first factor associated to the rotational symmetry and the second one with the taste symmetry. At non zero lattice spacing this invariance group is broken down to the isometries group of the lattice. Since we are looking at zero momentum states with a fixed quark number, staggered mesons can be classified according to their transformation properties under a subgroup of the RF group 3 , the so called geometric time slice group (GTS) that is generated by the spatial inversion $I_{s}$, the taste transformations $\Xi_{\mu}$ and the rotations of $\pi / 2$ angle $R^{k l}$. The staggered quark field $\chi(\mathbf{x})$ defined at the point $\mathbf{x}=(x, y, z, t)$ on a lattice with spacing $a$ forms an 8 dimensional irreducible representation (8irrep) of the GTS. Under the subgroup of the discretized version of the cubic rotation group $O_{h}$ it decomposes as $A_{1}^{+} \oplus A_{1}^{-} \oplus F_{1}^{+} \oplus F_{1}^{-}$where $A_{1}^{ \pm}$and $F_{1}^{ \pm}$are, respectively, the trivial and the three dimensional vector representation with parity \pm . To construct the meson states one has to take the tensor product of two staggered fields and just by counting one obtains sixty-four states that, following [14, we list in Table1. At a first sight the meson operators can be divided in four classes: the local operators $(n=1 . .4$ in Table 1) written as

$$
\mathcal{M}^{0-L}=\phi(\mathbf{x}) \bar{\chi}(\mathbf{x}) \chi(\mathbf{x}),
$$

with $\phi(\mathbf{x})$ being a phase factor depending on the choice of the channel, where the two staggered fields sit at the same lattice point; the one-link operators $(n=5 . .10)$ written schematically as

$$
\mathcal{M}^{1-L}=\phi(\mathbf{x}) \bar{\chi}(\mathbf{x}) \Delta_{i} \chi(\mathbf{x})
$$

where one has to introduce the shift operator as

$$
\Delta_{i} \chi(\mathbf{x})=1 / 2(\chi(\mathbf{x}+\hat{i})+\chi(\mathbf{x}-\hat{i}))
$$

and put the quark and the anti-quark in two next-neighbor lattice points; and finally the two and three link operators ( $n=11 . .16$ and $n=17 . .20$ respectively) defined as

$$
\mathcal{M}^{2-L}=\epsilon_{i j k} \phi(\mathbf{x}) \bar{\chi}(\mathbf{x}) \Delta_{i} \Delta_{j} \chi(\mathbf{x})
$$

${ }^{3}$ The rest frame group is the invariance group of the transfer matrix 


$$
\mathcal{M}^{3-L}=\phi(\mathbf{x}) \bar{\chi}(\mathbf{x}) \Delta_{1} \Delta_{2} \Delta_{3} \chi(\mathbf{x})
$$

where quark and anti-quark are separated by two and three links. More specifically, all the states can be classified according to the value $\boldsymbol{r}^{\sigma_{s}, \sigma_{123}}$ of the representation of GTS in which they lie, where $r$ is the dimension of the irrep., and $\sigma_{s}$ and $\sigma_{123}$ are, respectively, the eigenvalues of $I_{s}$, the spatial inversion, and of $X_{123}$, the parity under spatial taste inversion. Just for completeness, in the fourth column of Table 1 we have also indicated the meson description in the original language of Kogut and Susskind: using the spin $\otimes$ taste basis the staggered mesons can be written as $M=\bar{\psi}\left(\Gamma^{D} \otimes \Gamma^{T}\right) \psi$ where $\psi$ has four Dirac and four taste components and is defined on a coarse lattice with spacing $2 a$. From the fifth column one can read off the $J_{R}^{P C}$ quantum numbers of the lowest corresponding continuum states at zero temperature and finally in the last column the particle identification of those states is given.

At non-zero temperature the situation is different due to the fact that the temporal direction is special since it is related to the temperature through the relation $T=1 / a N_{\tau}$ where $N_{\tau}$ is the number of lattice points in that direction. For spatial correlation functions, which we are going to analyze, the subgroup of $O_{h}$ preserved in the zero temperature case is now broken since certain $\pi / 2$ rotations (for example in the $x-t$ and $y-t$ plane) are no more symmetries of the lattice discretization. As a consequence, the mesonic states have to be classified differently from Table 1. The new invariance subgroup is $D_{4}^{h}$, the discretized version of the cylinder symmetries with 16 elements divided into eight one-dimensional representations labeled by $A_{1}^{ \pm}, A_{2}^{ \pm}, B_{1}^{ \pm}, B_{2}^{ \pm}$and two two-dimensional irreps $E^{ \pm}$. Following $[29$ it is easy to understand how the irreps of $O_{h}$ reduce under $D_{4}^{h}$. Basically the one dimensional irrep of $O_{h}$ continues to be a 1-irrep of $D_{4}^{h}$ while the vector of $O_{h}$ decomposes into a singlet plus the two dimensional representation $E$. One can now do the tensor product of two staggered quark fields and find in which way the mesons transform under the invariance group at finite $T$. This has already been done by S. Gupta in 29 and summarizing his results we have that the three dimensional vector irreps, which in Table 1 are labeled by the numbers $n=3 . .9,11 . .14$, and 19,20 , split into a two plus a one dimensional representation. Concerning the tensor irreps. of dimension 6 , labeled by $n=9,10,15,16$, they split in four plus two dimensional representations.

If the temperature is further increased and approaches the infinite temperature limit, compared to the finite temperature case, more degeneracies will develop as in this limit the theory approaches the free case. Indeed to the extent that dimensional reduction is a good approximation, one sees an approximate $C_{v}^{4}$ symmetry, thus the representations $A_{1}^{+}, E^{+}, A_{1}^{-}$become degenerate with $A_{2}^{-}, E^{-}, A_{2}^{+}$ respectively and as a consequence the correlators fall into only very few multiplets.

In this work we study the local and non-local mesons built from $\bar{u} d, \bar{u} s$ and $\bar{s} s$ flavor combinations. In flavor singlet channels like e.g. $\bar{s} s$ only the quark-line connected part

\footnotetext{
${ }^{4}$ The symmetry group of the dimensionally reduced theory $C_{v}^{4} \simeq D_{4}$ is the group of the isometries of a square.
}

has been computed and the computationally demanding disconnected part has been neglected. The connected part of the staggered meson screening correlators, projected to zero transverse momentum, $\boldsymbol{p}_{\perp}=\left(p_{x}, p_{y}\right)$, and to zero (boson) Matsubara frequency $\omega_{n}$, are obtained as

$$
C(z)=\sum_{x, y, \tau} \sum_{\mathbf{v}, \mathbf{w} \in \mathfrak{L}} \phi(\boldsymbol{x})\left\langle\left(M_{\boldsymbol{x}+\mathbf{v}, \mathbf{0}}^{-1} U_{\mathbf{v}}(\mathbf{x})\right)\left(M_{\boldsymbol{x} \mathbf{w}}^{-1} U_{\mathbf{w}}(-\mathbf{w})\right)^{\dagger}\right\rangle,
$$

where $M_{\mathbf{0 x}}^{-1}$ is the full staggered quark propagator from $\mathbf{0}$ to $\boldsymbol{x}, \phi(\mathbf{x})$ is a phase depending on the choice of the channel and $\mathfrak{L}=\{0\}$ for the local correlators, $\mathfrak{L}=\{ \pm \hat{i}\}$ for the one-link correlators and is equal to $\{ \pm \hat{i} \pm \hat{j}\}$ and $\{ \pm \hat{x} \pm \hat{y} \pm \hat{\tau}\}$ respectively for the two-link and the three link correlation functions 5 . Thus while for the computation of the screening mass of the local meson only one inversion of the fermion matrix is needed, for the one-link, two-link and three-link operators we need three, five and nine inversions respectively that make the computation more demanding compared to the local operators. Since a staggered fermion meson correlator, in general, contains two different mesons with opposite parity [14] one has to parametrize it as

$$
\begin{aligned}
C(z)= & A_{N O} \cosh \left[M_{-}\left(z-\frac{N_{s}}{2}\right)\right] \\
& -(-1)^{z} A_{O} \cosh \left[M_{+}\left(z-\frac{N_{s}}{2}\right)\right]
\end{aligned}
$$

According to our phase convention, for the local and twolink operators $M_{-}\left(M_{+}\right)$corresponds to the screening mass of the lightest negative (positive) parity state and comes as the non-oscillating, NO, (oscillating, O,) part of the screening correlator and viceversa for the one and three-link operators.

\section{Staggered Chiral Perturbation Theory}

In this section we review some elements of staggered chiral perturbation theory $(\mathrm{S} \chi \mathrm{PT})$ and more in detail its prediction regarding taste symmetry violation. The chiral lagrangian of the $\mathrm{S} \chi \mathrm{PT}$ has been introduced in 23 for a single staggered flavor and generalized to multiple flavors in [24,26]. It describes the low energy dynamics of pseudoGoldstone bosons near the continuum and chiral limit and provides a systematic method to discuss the consequences of chiral symmetry breaking in the framework of the staggered formulation of lattice QCD.

Starting with an $S U(3)$ gauge theory with $n$ flavors of staggered fermions, in the combined chiral-continuum limit the theory possesses an $S U(4 n)_{L} \times S U(4 n)_{R}$ chiral symmetry which is spontaneously broken to $S U(4 n)_{V}$. The $16 n^{2}-1$ mesonic fields that arise from the breaking can be collected into a unitary matrix

$$
\Sigma=\exp \left(i \Phi / f_{\pi}\right)
$$

\footnotetext{
$5 \hat{i}$ and $\hat{j} \in\{\hat{x}, \hat{y}, \hat{\tau}\}$

6 Note that both amplitudes are positive 31, $A_{N O}, A_{O} \geq 0$
} 


\begin{tabular}{|c|c|c|c|c|c|}
\hline $\mathrm{N}$ & Operator & $r^{\sigma_{s} \sigma_{123}}$ & $\Gamma^{D} \times \Gamma^{F}$ & State & Particle \\
\hline \multirow[t]{2}{*}{1} & $\bar{\chi} \chi$ & $1^{++}$ & $1 \otimes 1$ & $0_{S}^{++}$ & $f_{0}$ \\
\hline & & & $\gamma_{4} \gamma_{5} \otimes \xi_{4} \xi_{5}$ & $0_{A}^{-+}$ & $\pi$ \\
\hline \multirow[t]{2}{*}{2} & $\eta_{4} \zeta_{4} \bar{\chi} \chi$ & $1^{+-}$ & $\gamma_{4} \otimes \xi_{4}$ & $0_{A}^{+-}$ & - \\
\hline & & & $\gamma_{5} \otimes \xi_{5}$ & $0_{A}^{-+}+$ & $\pi$ \\
\hline \multirow[t]{2}{*}{3} & $\eta_{i} \epsilon \zeta_{i} \bar{\chi} \chi$ & $3^{+-}$ & $\gamma_{i} \gamma_{5} \otimes \xi_{i} \xi_{5}$ & $1_{A}^{++}$ & $a_{1}$ \\
\hline & & & $\gamma_{i} \gamma_{4} \otimes \xi_{i} \xi_{4}$ & $1_{A}^{--}$ & $\rho$ \\
\hline \multirow[t]{2}{*}{4} & $\eta_{4} \zeta_{4} \eta_{i} \epsilon \zeta_{i} \bar{\chi} \chi$ & $3^{++}$ & $\gamma_{j} \gamma_{k} \otimes \xi_{j} \xi_{k}$ & $1_{A}^{+-}$ & $b_{1}$ \\
\hline & & & $\gamma_{i} \otimes \xi_{i}$ & $1_{A}^{--}$ & $\rho$ \\
\hline \multirow[t]{2}{*}{5} & $\bar{\chi} \eta_{i} \Delta_{i \chi}$ & $3^{-+}$ & $\gamma_{i} \otimes 1$ & $1_{S}^{-}-$ & $\omega$ \\
\hline & & & $\gamma_{j} \gamma_{k} \otimes \xi_{4} \xi_{5}$ & $1_{A}^{+-}$ & $b_{1}$ \\
\hline \multirow[t]{2}{*}{6} & $\eta_{4} \zeta_{4} \bar{\chi} \eta_{i} \Delta_{i} \chi$ & $3^{--}$ & $\gamma_{i} \gamma_{4} \otimes \xi_{4}$ & $1_{A}^{--}$ & $\rho$ \\
\hline & & & $\gamma_{i} \gamma_{5} \otimes \xi_{5}$ & $1_{A}^{++}$ & $a_{1}$ \\
\hline \multirow[t]{2}{*}{7} & $\bar{\chi} \epsilon \zeta_{i} \Delta_{i} \chi$ & $3^{--}$ & $\gamma_{5} \otimes \xi_{i} \xi_{5}$ & $0_{A}^{-+}+$ & $\pi$ \\
\hline & & & $\gamma_{4} \otimes \xi_{i} \xi_{4}$ & $0_{A}^{+-}$ & - \\
\hline \multirow[t]{2}{*}{8} & $\eta_{4} \zeta_{4} \bar{\chi} \epsilon \zeta_{i} \Delta_{i} \chi$ & $3^{-+}$ & $\gamma_{4} \gamma_{5} \otimes \xi_{j} \xi_{k}$ & $0_{A}^{-+}+$ & $\pi$ \\
\hline & & & $1 \otimes \xi_{i}$ & $0_{A}^{++}$ & $a_{0}$ \\
\hline \multirow[t]{2}{*}{9} & $\eta_{i} \epsilon \zeta_{i} \bar{\chi} \zeta_{j} \Delta_{j} \chi$ & $6^{--}$ & $\gamma_{k} \gamma_{4} \otimes \xi_{i} \xi_{5}$ & $1_{A}^{--}$ & $\rho$ \\
\hline & & & $\gamma_{k} \gamma_{5} \otimes \xi_{i} \xi_{4}$ & $1_{A}^{++}$ & $a_{1}$ \\
\hline \multirow[t]{2}{*}{10} & $\eta_{4} \zeta_{4} \eta_{i} \epsilon \zeta_{i} \bar{\chi} \zeta_{j} \Delta_{j} \chi$ & $6^{-+}$ & $\gamma_{k} \otimes \xi_{j} \xi_{k}$ & $1_{A}^{--}$ & $\rho$ \\
\hline & & & $\gamma_{i} \gamma_{j} \otimes \xi_{i}$ & $1_{A}^{+-}$ & $b_{1}$ \\
\hline \multirow[t]{2}{*}{11} & $\epsilon_{i j k} \bar{\chi} \eta_{i} \Delta_{i}\left(\eta_{j} \Delta_{j} \chi\right)$ & $3^{++}$ & $\gamma_{i} \gamma_{j} \otimes 1$ & $1_{S}^{++}$ & $h_{1}$ \\
\hline & & & $\gamma_{k} \otimes \xi_{4} \xi_{5}$ & $1_{A}^{--}$ & $\rho$ \\
\hline \multirow[t]{2}{*}{12} & $\epsilon_{i j k} \eta_{4} \zeta_{4} \bar{\chi} \eta_{i} \Delta_{i}\left(\eta_{j} \Delta_{j} \chi\right)$ & $3^{+-}$ & $\gamma_{k} \gamma_{5} \otimes \xi_{4}$ & $1_{A}^{++}+$ & $a_{1}$ \\
\hline & & & $\gamma_{k} \gamma_{4} \otimes \xi_{5}$ & $1_{A}^{-}-$ & $\rho$ \\
\hline \multirow[t]{2}{*}{13} & $\epsilon_{i j k} \bar{\chi} \zeta_{i} \Delta_{i}\left(\zeta_{j} \Delta_{j} \chi\right)$ & $3^{++}$ & $1 \otimes \xi_{i} \xi_{j}$ & $0_{A}^{++}$ & $a_{0}$ \\
\hline & & & $\gamma_{4} \gamma_{5} \otimes \xi_{k}$ & $0_{A}^{-+}$ & $\pi$ \\
\hline \multirow[t]{2}{*}{14} & $\epsilon_{i j k} \eta_{4} \zeta_{4} \bar{\chi} \zeta_{i} \Delta_{i}\left(\zeta_{j} \Delta_{j} \chi\right)$ & $3^{+-}$ & $\gamma_{4} \otimes \xi_{k} \xi_{5}$ & $0_{A}^{+-}$ & - \\
\hline & & & $\gamma_{5} \otimes \xi_{k} \xi_{4}$ & $0_{A}^{-+}+$ & $\pi$ \\
\hline \multirow[t]{2}{*}{15} & $\eta_{k} \zeta_{k} \bar{\chi} \eta_{i} \Delta_{i}\left(\zeta_{j} \Delta_{j} \chi\right)$ & $6^{++}$ & $\gamma_{i} \gamma_{k} \otimes \xi_{j} \xi_{k}$ & $1_{A}^{+-}$ & $b_{1}$ \\
\hline & & & $\gamma_{j} \otimes \xi_{i}$ & $1_{A}^{--}$ & $\rho$ \\
\hline \multirow[t]{2}{*}{16} & $\eta_{4} \zeta_{4} \eta_{k} \zeta_{k} \bar{\chi} \eta_{i} \Delta_{i}\left(\zeta_{j} \Delta_{j} \chi\right)$ & $6^{+-}$ & $\gamma_{j} \gamma_{5} \otimes \xi_{i} \xi_{5}$ & $1_{A}^{++}$ & $a_{1}$ \\
\hline & & & $\gamma_{j} \gamma_{4} \otimes \xi_{i} \xi_{4}$ & $1_{A}^{--}$ & $\rho$ \\
\hline \multirow[t]{2}{*}{17} & $\bar{\chi} \eta_{1} \Delta_{1}\left(\eta_{2} \Delta_{2}\left(\eta_{3} \Delta_{3} \chi\right)\right)$ & $1^{-+}$ & $\gamma_{4} \gamma_{5} \otimes 1$ & $0_{S}^{-+}$ & $\eta^{\prime}$ \\
\hline & & & $1 \otimes \xi_{4} \xi_{5}$ & $0_{A}^{++}$ & $a_{0}$ \\
\hline \multirow[t]{2}{*}{18} & $\eta_{4} \zeta_{4} \bar{\chi} \eta_{1} \Delta_{1}\left(\eta_{2} \Delta_{2}\left(\eta_{3} \Delta_{3} \chi\right)\right)$ & $1^{--}$ & $\gamma_{5} \otimes \xi_{4}$ & $0_{A}^{-+}+$ & $\pi$ \\
\hline & & & $\gamma_{4} \otimes \gamma_{5}$ & $0_{A}^{+-}$ & - \\
\hline \multirow[t]{2}{*}{19} & $\eta_{i} \epsilon \zeta_{i} \bar{\chi} \eta_{1} \Delta_{1}\left(\eta_{2} \Delta_{2}\left(\eta_{3} \Delta_{3} \chi\right)\right)$ & $3^{--}$ & $\gamma_{i} \gamma_{4} \otimes \xi_{i} \xi_{5}$ & $1_{A}^{--}$ & $\rho$ \\
\hline & & & $\gamma_{i} \gamma_{5} \otimes \xi_{i} \xi_{4}$ & $1_{A}^{++}$ & $a_{1}$ \\
\hline \multirow[t]{2}{*}{20} & $\eta_{4} \zeta_{4} \eta_{i} \epsilon \zeta_{i} \bar{\chi} \eta_{1} \Delta_{1}\left(\eta_{2} \Delta_{2}\left(\eta_{3} \Delta_{3} \chi\right)\right)$ & $3^{-+}$ & $\gamma_{i} \otimes \xi_{j} \xi_{k}$ & $1_{A}^{--}$ & $\rho$ \\
\hline & & & $\gamma_{j} \gamma_{k} \otimes \xi_{i}$ & $1_{A}^{+-}$ & $b_{1}$ \\
\hline
\end{tabular}

Table 1. Irreducible representations and their operators for mesonic states. Here $\Delta_{i} f(x, t)=\frac{1}{2}[f(x+\hat{i}, t)+f(x-\hat{i}, t)]$ while $\eta_{\mu}(x)=(-1)^{x_{1}+\ldots x_{\mu-1}}, \zeta_{\mu}(x)=(-1)^{x_{\mu+1}+\ldots x_{4}}$ and $\epsilon(x)=(-1)^{x_{1}+\ldots x_{4}}$.

with

$$
\Phi=\left(\begin{array}{cccc}
U & \pi^{+} & K^{+} & \ldots \\
\pi^{-} & D & K^{0} & \cdots \\
K^{-} & \bar{K}^{0} & S & \cdots \\
\vdots & \vdots & \vdots & \ddots
\end{array}\right)
$$

$$
T_{a}=\xi_{5}, i \xi_{\mu 5}, i \xi_{\mu \nu}, \xi_{\mu}, \xi_{I} .
$$

and $f_{\pi}$ is the pion decay constant. Using the usual powercounting scheme to derive the staggered chiral lagrangian

$$
p^{2} / \Lambda_{Q C D}^{2} \approx m / \Lambda_{Q C D} \approx a^{2} \Lambda_{Q C D}^{2}
$$

where $U=\sum_{a=1}^{16} U_{a} T_{a}, \pi^{+}=\sum_{a=1}^{16} \pi_{a}^{+} T_{a}$, etc. are $4 \times 4$ one obtains to lowest order submatrices expressed in the Hermitian basi: 7

\footnotetext{
${ }^{7} \xi_{\mu}$ are the Dirac gamma matrices and $\xi_{I}=\mathbb{I}_{4 \times 4}$
}

$$
\mathcal{L}_{S \chi P T}=\frac{f_{\pi}^{2}}{8} \operatorname{Tr}\left(\partial_{\mu} \Sigma \partial_{\mu} \Sigma^{\dagger}\right)-\frac{\mu f_{\pi}^{2}}{4} \operatorname{Tr}\left(M^{\dagger} \Sigma+M \Sigma^{\dagger}\right)+
$$




$$
\frac{2}{3} m_{0}^{2}\left(U_{I}^{2}+D_{I}^{2}+S_{I}^{2}+\cdots\right)+a^{2} V(\Sigma)
$$

where

$$
M=\left(\begin{array}{cccc}
m_{u} \mathbb{I} & 0 & 0 & \cdots \\
0 & m_{d} \mathbb{I} & 0 & \cdots \\
0 & 0 & m_{s} \mathbb{I} & \cdots \\
\vdots & \vdots & \vdots & \ddots
\end{array}\right)
$$

is the quark mass matrix, $m_{0}$ models the effect of the anomaly and $V(\Sigma)$ is the taste-breaking potential. This potential arises from the four-fermion operators in the quark effective action and is a linear combination of operators

$$
-V(\Sigma)=\sum_{i} C_{i} O_{i}
$$

with $C_{i}$ six unknown low energy effective constants (LECs). More in detail the operators that enter in the potential read as

$$
\begin{gathered}
O_{1}=\operatorname{Tr}\left(\Xi_{5} \Sigma \Xi_{5} \Sigma^{\dagger}\right) \\
O_{2 V}=\frac{1}{4} \sum_{\mu}\left[\operatorname{Tr}\left(\Xi_{\mu} \Sigma\right) \operatorname{Tr}\left(\Xi_{\mu} \Sigma\right)+h . c .\right] \\
O_{2 A}=\frac{1}{4} \sum_{\mu}\left[\operatorname{Tr}\left(\Xi_{\mu 5} \Sigma\right) \operatorname{Tr}\left(\Xi_{\mu 5} \Sigma\right)+h . c .\right] \\
O_{3}=\frac{1}{2} \sum_{\mu}\left[\operatorname{Tr}\left(\Xi_{\mu} \Sigma \Xi_{\mu} \Sigma\right)+h . c .\right] \\
O_{4}=\frac{1}{2} \sum_{\mu}\left[\operatorname{Tr}\left(\Xi_{\mu 5} \Sigma \Xi_{\mu 5} \Sigma\right)+h . c .\right] \\
O_{5 V}=\frac{1}{2} \sum_{\mu}\left[\operatorname{Tr}\left(\Xi_{\mu} \Sigma\right) \operatorname{Tr}\left(\Xi_{\mu} \Sigma^{\dagger}\right)+h . c .\right] \\
O_{5 A}=\frac{1}{2} \sum_{\mu}\left[\operatorname{Tr}\left(\Xi_{\mu 5} \Sigma\right) \operatorname{Tr}\left(\Xi_{\mu 5} \Sigma^{\dagger}\right)+\text { h.c. }\right] \\
O_{6}=\frac{1}{2} \sum_{\mu<\nu} \operatorname{Tr}\left(\Xi_{\mu \nu} \Sigma \Xi_{\mu \nu} \Sigma^{\dagger}\right)
\end{gathered}
$$

Here the $4 n \times 4 n$ matrices $\Xi_{T}$ are just the generalizations of the $4 \times 4$ taste matrices $\xi_{T}$. If we focus only on the non-diagonal flavor meson 8 it is sufficient to expand the lagrangian to quadratic order in the mesonic fields and consider only the single-trace terms. ¿From the expansion one can read off the tree-level masses of the mesons :

$$
m_{M_{B}}^{2}=\mu\left(m_{a}+m_{b}\right)+a^{2} \Delta_{\xi_{B}}
$$

where the meson $M$ is composed of two quarks $a$ and $b$ and where

$$
\Delta\left(\xi_{5}\right)=\Delta_{P S}=0
$$

\footnotetext{
8 Simulations in which disconnected contributions are not taken into account describe only non-diagonal flavor states $\pi^{ \pm}, K^{ \pm}$..
}

$$
\begin{array}{r}
\Delta\left(\xi_{\mu 5}\right)=\Delta_{A}=\frac{16}{f_{\pi}^{2}}\left(C_{1}+3 C_{3}+C_{4}+3 C_{6}\right) \\
\Delta\left(\xi_{\mu \nu}\right)=\Delta_{T}=\frac{16}{f_{\pi}^{2}}\left(2 C_{3}+2 C_{4}+4 C_{6}\right) \\
\Delta\left(\xi_{\mu}\right)=\Delta_{V}=\frac{16}{f_{\pi}^{2}}\left(C_{1}+C_{3}+3 C_{4}+3 C_{6}\right) \\
\Delta\left(\xi_{I}\right)=\Delta_{I}=\frac{16}{f_{\pi}^{2}}\left(4 C_{3}+4 C_{4}\right)
\end{array}
$$

For the flavor neutral mesons $(U, D, \cdots)$ the situation is more complicated since in the expansion of the two-trace operators some two-point vertices mixing the taste-vector, taste-axial and taste-singlet flavor-neutral states appear and thus the Lagrangian receives a contribution of the form

$$
\begin{array}{r}
\mathcal{L}=-\frac{16 a^{2}}{f_{\pi}^{2}} \delta_{V}\left(U_{\xi_{\mu}}+D_{\xi_{\mu}}+\cdots\right)^{2}+ \\
-\frac{16 a^{2}}{f_{\pi}^{2}} \delta_{A V}\left(U_{\xi_{\mu 5}}+D_{\xi_{\mu 5}}+\cdots\right)^{2} \\
-\frac{16 a^{2}}{f_{\pi}^{2}} \delta_{I}\left(U_{I}+D_{I}+\cdots\right)^{2}
\end{array}
$$

For more details about the taste splitting in such channels we refer to 24 .

One interesting thing to note is that the symmetry group of the lattice theory at $\mathcal{O}\left(a^{2}\right)$ is enlarged in the meson sector since the potential $\mathcal{V}(\Sigma)$ is rotationally and $S O(4)$ taste invariant. The consequence of this enhancement of symmetry is that the sixteen taste states instead of falling into eight different irreps of the GTS as predicted from group theory split into only five degenerate multiplets according to the $S O(4)$ symmetry (eq. 23]27). The vanishing of $\Delta\left(\xi_{5}\right)$ is due to the taste non-singlet $U_{\epsilon}(1)$ symmetry which is unbroken by the lattice regulator, making the $\gamma_{5} \times \xi_{5}$ meson a true Goldstone boson. This prediction of the splitting has been well confirmed in a series of lattice simulations [15, 16, 17, 27.

This analysis is strictly valid at temperature zero. Since our aim is to study the taste symmetry violations at finite $T$ we need to understand how the predictions of $\mathrm{S} \chi \mathrm{PT}$ get modified. In particular two scenarios seem possible. The first one is that the formal structure of the chiral Lagrangian remains the same as in (12) but the coefficients $C_{i}$ and $f_{\pi}$ acquire a temperature dependence. At least this hypothesis could work until $T_{c}$ where the chiral symmetry is restored and chiral perturbation theory breaks down. Indeed, as we will see, in the vicinity of the transition the value of the pion decay constant that "measures" the strength of the chiral symmetry breaking goes to zero rapidly. A second scenario can be described by a different lagrangian since in principle operators different from the $T=0$ case are admitted in the Symanzik effective theory. This is due to the fact that, as explained in the previous section, the invariance group of the zero temperature theory is broken down to a subgroup. 


\begin{tabular}{|ll|lr|rr|}
\hline & & \multicolumn{2}{|c|}{$24^{3} \times 6$} & \multicolumn{2}{c|}{$32^{3} \times 8$} \\
$\beta$ & $m_{l}$ & $T$ & conf. & $T$ & conf. \\
\hline 3.351 & 0.00591 & 145 & 695 & & \\
3.410 & 0.00412 & 175 & 786 & & \\
3.430 & 0.00370 & 186 & 875 & 139 & 793 \\
3.445 & 0.00344 & 197 & 946 & & \\
3.455 & 0.00329 & 200 & 405 & & \\
3.460 & 0.00313 & 203 & 729 & & \\
3.490 & 0.00290 & 226 & 775 & & \\
3.500 & 0.00253 & & & 175 & 614 \\
3.510 & 0.00259 & 240 & 577 & & \\
3.530 & 0.00253 & & & 192 & 590 \\
3.540 & 0.00240 & 259 & 591 & & \\
3.570 & 0.00212 & 281 & 525 & 211 & 738 \\
3.585 & 0.00192 & & & 219 & 360 \\
3.630 & 0.00170 & 326 & 389 & & \\
3.690 & 0.00150 & 365 & 448 & & \\
3.760 & 0.00130 & 424 & 465 & 318 & 588 \\
3.820 & 0.00125 & & & 361 & 488 \\
3.920 & 0.00110 & & & 410 & 481 \\
3.920 & 0.00092 & 532 & 480 & & \\
4.000 & 0.00092 & & & 475 & 493 \\
4.080 & 0.00081 & & & 549 & 392 \\
\hline
\end{tabular}

Table 2. Coupling constants $\beta$ and light quark masses $m_{l}$, temperatures $T$ in $\mathrm{MeV}$ and the number of configurations of the given sizes on which screening masses were computed. The strange quarks have always been choice as $m_{s}=10 m_{l}$.

\section{Results}

In order to perform this analysis we have used dynamical $N_{f}=2+1$ gauge field configurations generated with the RHMC algorithm 32 by the RBC-Bielefeld 30,33 and the HotQCD 34] collaborations using the p4 staggered action. The configurations were generated along the Line of Constant Physics (LCP) obtained by tuning the bare quark masses such that at zero temperature the (Goldstone) pion mass $m_{\pi}$ is approximately $220 \mathrm{MeV}$ and the kaon mass $m_{K}$ equals $500 \mathrm{MeV}$. The bare strange quark mass has always been 10 times larger than the light one, $m_{s}=10 m_{l}$. In table (2), for both lattices $24^{3} \times 6$ and $32^{3} \times 8$ used in the simulations, the values of the coupling constant and of the bare light quark mass at which the configurations were generated are reported. The number of configurations analyzed was about 500-600 at each temperature, separated by 10 time units. For more details about the simulations and scale settings we refer to 33 . In the following we report on our results for the screening masses of the local and non-local staggered mesons extracted from the lattice calculation of the spatial correlators in some of the twenty mesonic channels listed in Table (1). More in detail we focus on the pion channels labeled with $n=1,2,7,8,13,14,17,18$ since we are primarily interested in the taste splittings in this multiplet. However our analysis is not limited to that but we also inspected some scalar, vector and axial-vector channels to extract some other useful information from them, for example about the restoration of the chiral symmetry and the finite temperature splittings of those multiplets.
Before starting the presentation of the numerical results we recall that a staggered correlator contains two different mesons with opposite parity (see eq. 8). This is valid in general except for the channels $2,7,14,18$ where only the pion contributes. From these channels we are able to extract the screening masses above and below the transition fitting as usual the correlators with the exponential functions and with errors determined by jack-knifing the fit. In the other channels the pions have to be extracted from the oscillating $(n=1,13)$ or the non-oscillating part ( $n=8,17)$ but also the scalars contribute to the correlators. If one performs the analysis at zero temperature no problem occurs in the determination of the screening masses of all pion taste components. Unfortunately the situation is quite different at finite temperature since in the channels $n=1,8,13,17$ the amplitudes of the pion states die out very fast with rising temperature and as a consequence we are not able to extract their screening masses. Indeed at temperatures above the transition the pion contributions to the correlators disappear completely and the channels are dominated by the scalars. The lack of these screening mass data makes the comparison with the $\mathrm{S} \chi \mathrm{PT}$ incomplete. Differently from the $T=0$ case in which one can check an $S O(4)$ restoration of the taste symmetry predicted by the $\mathrm{S} \chi \mathrm{PT}$, we are not able to verify if an analogous enhancement happens also at finite temperature.

For the axial-vector and vector channels the situation is not so clear, we have seen in all analyzed channels the presence of both parity states but, likewise to what happens in the local sector [35, using the point sources we were able to extract only few values of the screening mass for very high temperatures (above $2 T_{c}$ ) since the correlators are very noisy.

In addition to the investigation done for mesons made out off two degenerate $u$ and $d$ quarks we have also performed some calculations for meson with flavor content $\bar{u} s$ and $\bar{s} s$ to compare the result with the light sector and extract some additional checks regarding the $\mathrm{S} \chi \mathrm{PT}$ that predicts the flavor independence of the taste violation.

Let us start with figure 1 where we present the data on the screening mass of the various taste components of the pion multiplet labeled by their taste matrix $[\xi]$. In particulari, in the pictures 1(a) and 1(c) we plot the screening masses in units of $r_{0}$ as function of the temperature at $N_{\tau}=8$ and $N_{\tau}=6$ respectively. For both lattices we can observe that below the transition the Goldstone boson screening mass $\left([\xi]=\xi_{5}\right)$ remains approximatively constant while for the non local operators a slight decrease of their masses occurs below $T_{c}$. Above the transition there is a rapid linear rise of the values of the masses for all the states with increasing temperature. From the figures $1(\mathrm{~b})$ and $1(\mathrm{~d})$ where the screening masses in unit of $T$ as function of the temperature are plotted one can note a strong decrease for the non-local operator masses below the transition and an increase above that point with an approach from below to the free continuum result given by $2 \pi T$ (indicated by the continuum line) where the taste symmetry is restored completely. 


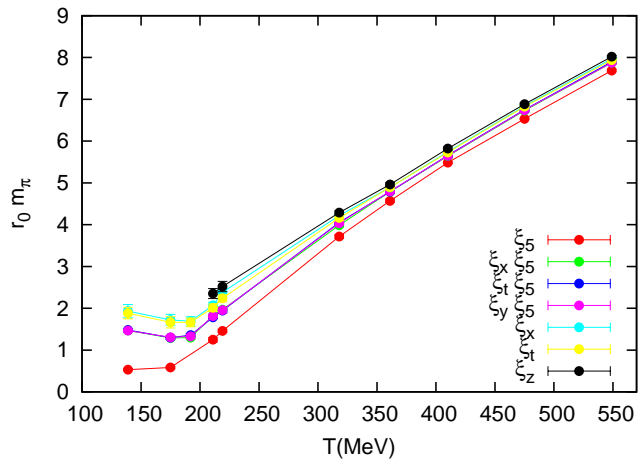

(a)

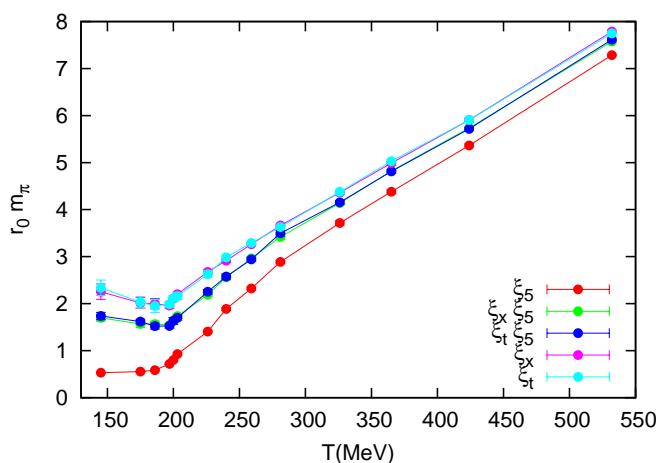

(c)

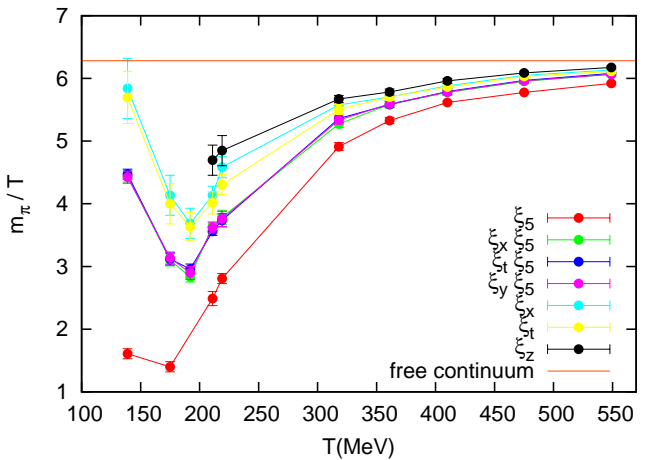

(b)

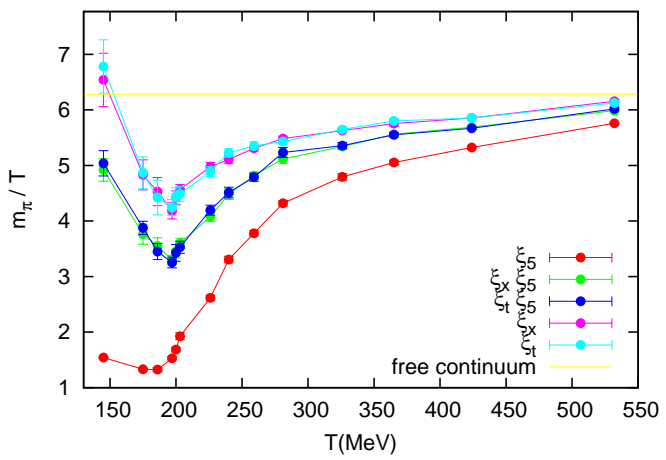

(d)

Fig. 1. Temperature dependence of the pseudoscalar screening mass $\left(\gamma_{5} \otimes[\xi]\right)$ in units of $r_{0}$ and of the temperature at $N_{\tau}=8$ $(a, b)$ and $N_{\tau}=6(c, d)$ for different components of the multiplet identified by the taste matrix $[\xi]$.

The scalar multiplet is shown in fig. 2 where we plot the temperature dependence of the screening masses of the various taste components normalized by $r_{0} 2(\mathrm{a})$. $2(\mathrm{c})$ and by the temperature $2(\mathrm{~b}), 2(\mathrm{~d})]$. Below the transition it is difficult to obtain quantitative results since the correlators turn out to be very noisy in this region, especially for the non-local observables. At temperature above $1.2 T_{c}$ all scalar correlators are degenerate with the pseudoscalar ones in corresponding taste channels.

In figure 3 we show the flavor dependence of the screening mass for the local and the 1-link pseudoscalar channel. More precisely, we have calculated and plotted the screening masses in the light $(\bar{u} d)$, light-strange $(\bar{u} s)$ and strange $(\bar{s} s)$ sectors. While above $1.5 T_{c}$ for both, local and 1-link operator no difference between the three sectors can be observed since the quark mass term becomes irrelevant, below $T_{c}$ Pion, Kaon and $\phi$ split as expected. Furthermore, in the region below the transition we cannot identify any substantial temperature effect on the local operator's masses while for the one-link operators a slight decrease can be observed as the temperature is increased until $T_{c}$. Note however that this data has been obtained at fixed $N_{\tau}$.
In the following we will discuss the taste splittings which are defined as the differences of squared pseudoscalar masses of different taste, see Eq.(22),

$$
\Delta_{\xi}=m_{\xi}^{2}-m_{\xi_{5}}^{2}
$$

and are traditionally but somewhat arbitrarily normalized to $(200 \mathrm{MeV})^{2}$. In order to understand the effect of the temperature on the taste symmetry violation in the group of figures (41) we have plotted the taste splittings as function of $T$ at fixed $\beta$ values. The results indicate that while in the confined phase there is essentially no effect of the temperature on the taste splitting, as one can figure out from figure 4(a), above the transition a temperature effect can be clearly read from 4(b) 4(c) 4(d) and 4(e). This unexpected behavior above $T_{c}$ deserves some comments that we postpone to the discussion section. Here we underline the fact and note that this effect seems independent of the lattice spacing since the slope of the increase stays approximatively constant for the configurations at different beta values.

In the group of figures 5 we study the lattice spacing dependence of the taste violation at fixed temperature, plotting the value of the taste splitting as function of $a^{2}$. For 


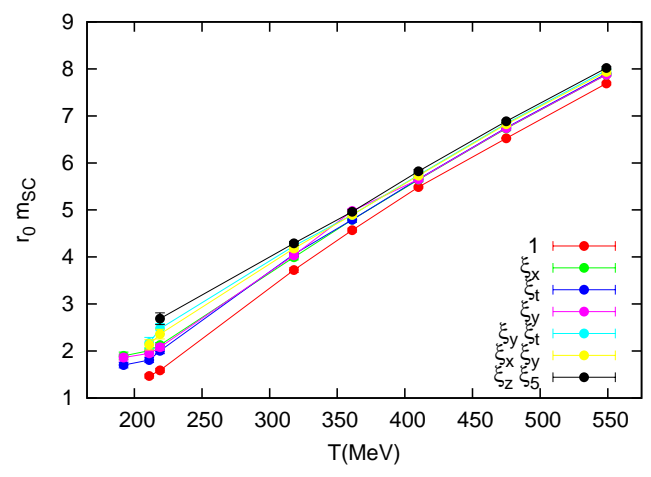

(a)

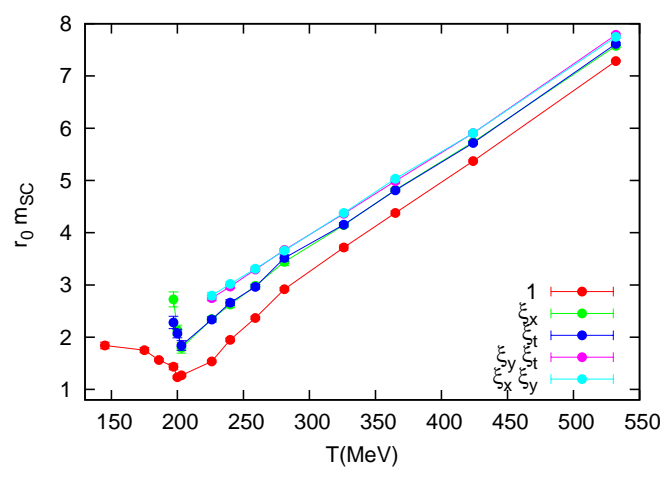

(c)

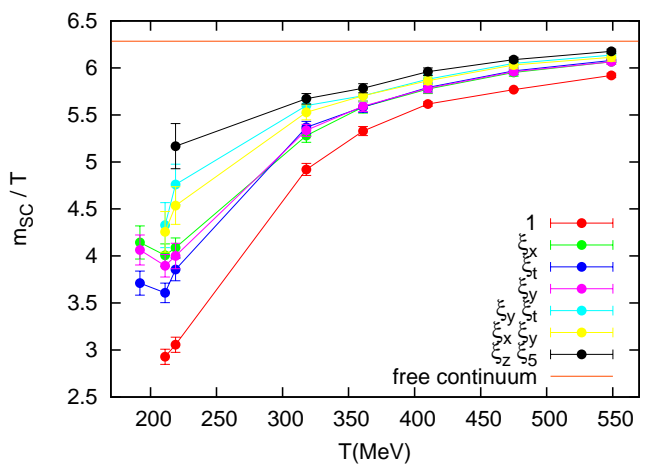

(b)

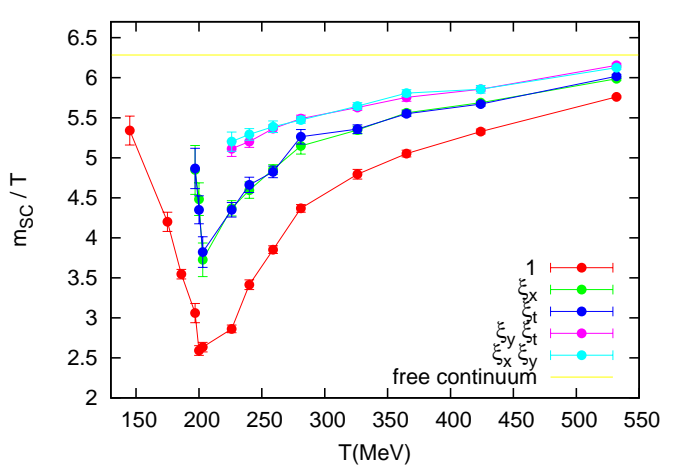

(d)

Fig. 2. Temperature dependence of the scalar screening mass $(1 \otimes[\xi])$ in unit of $r_{0}$ and of the temperature at $N_{\tau}=8(\mathrm{a}, \mathrm{b})$ and $N_{\tau}=6(\mathrm{c}, \mathrm{d})$ for different components of the multiplet identified by the taste matrix $[\xi]$.

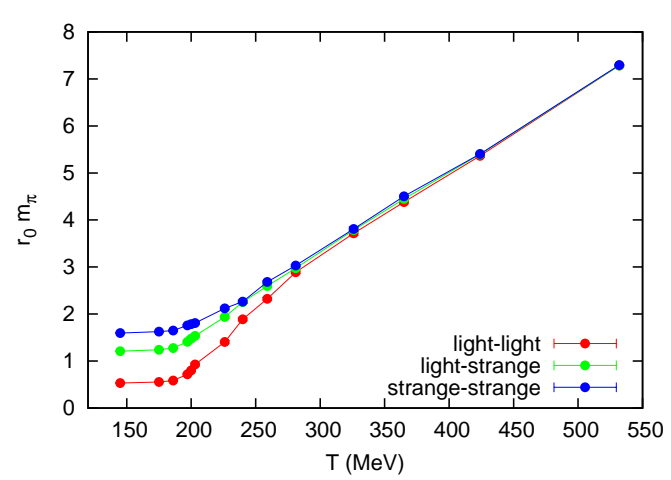

(a)

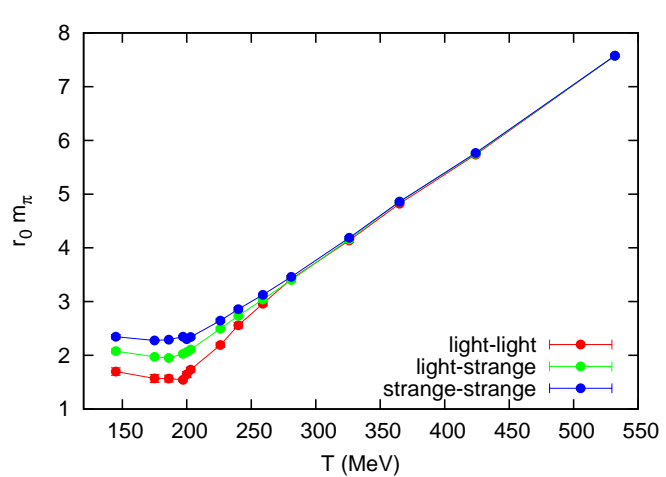

(b)

Fig. 3. (a) Flavor dependence of the screening mass for the local pseudoscalar operator $\left(\gamma_{5} \otimes \xi_{5}\right)$ in units of $r_{0}$ at $N_{\tau}=6$. (b) Flavor dependence of the screening mass for the 1-link pseudoscalar operator $\left(\gamma_{5} \otimes \xi_{x} \xi_{5}\right)$ in units of $r_{0}$ at $N_{\tau}=6$. 


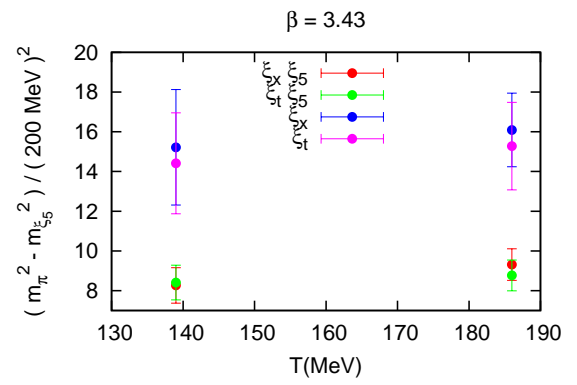

(a)

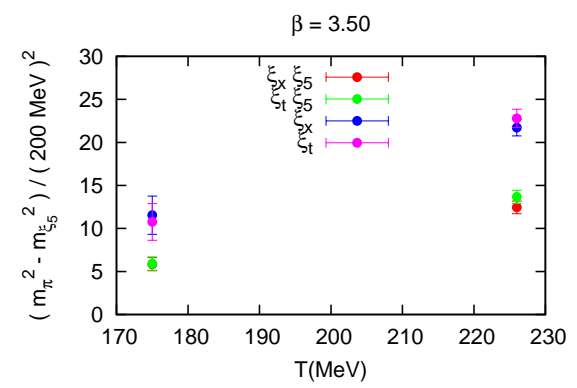

(b)

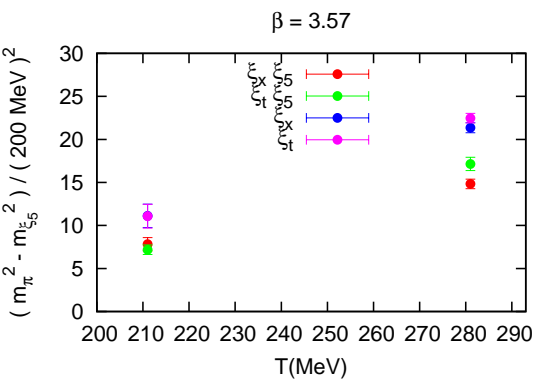

(c)

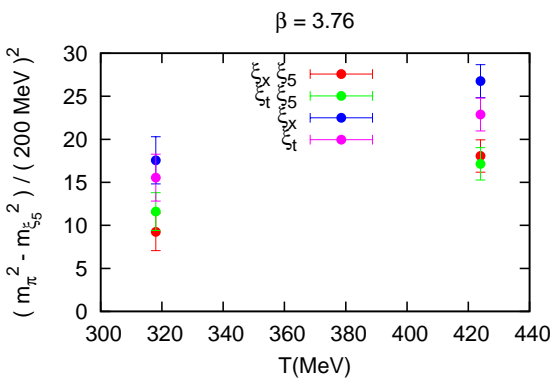

(d)

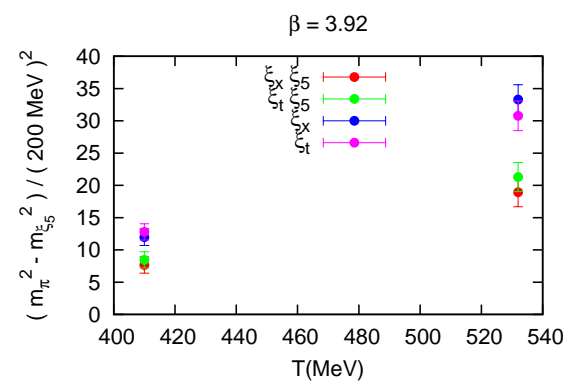

(e)

Fig. 4. Effect of the temperature on the taste violation at fixed $\beta$ values : (a) $\beta=3.43$, (b) $\beta=3.50$, (c) $\beta=3.57$, (d) $\beta=3.76$, (e) $\beta=3.92$

all analyzed temperatures the taste splittings are compatible with an $a^{2}$ behavior. However as we can easily see from 6(a) and 6(b) while below the transition the slope is more or less constant above $T_{c}$ its values start to increase with the temperature. This is an indication that in the low temperature regime the predictions of the $\mathrm{S} \chi \mathrm{PT}$ on the taste splitting seem to be valid and independent from $T$; even if above the transition these predictions are no more reliable an $a^{2}$ behavior is still observed but the temperature starts to affect the taste violation making the quantity $\Delta_{[\xi]}$ temperature-dependent.

In figure $7(\mathrm{a})$ we have plotted the taste splitting for different taste states in units of $200 \mathrm{MeV}$ as function of the temperature. The dependence of the taste violation cannot be extracted trivially looking at this pictures since in the plot we are comparing different finite temperature configurations with the same $N_{\tau}$ but thus with different values of the lattice spacing. For example comparing this figure with 6(a) and 6(b) one can note that the strong decreasing of the taste splitting observed below $T_{c}$ in $7(\mathrm{a})$ and $\left(7(\mathrm{~b})\right.$ is just a pure discretization effect. Above $\overline{T_{c}}$ the behavior of the splittings changes drastically, first starting to rise rapidly and at $1.5 T_{c}$ there is a flattening of the slope but again this is a combined lattice spacing and temperature effect.

The flavor dependence on the taste violation is analyzed in the figure $7(\mathrm{~b})$ where the quantity $\Delta_{\xi_{x}}=m_{\gamma_{5} \otimes \xi_{x}}^{2}-m_{\gamma_{5} \otimes \gamma_{5}}^{2}$ for the $\bar{u} d, \bar{u} s$ and $\bar{s} s$ channel is plotted. In the range of the temperature analyzed the screening masses in the different sectors are in agreement within the error bars. But while below the transition where the quark mass is relevant this fact is a not-trivial prediction of the staggered chiral perturbation theory, above $1.5 T_{c}$ the plot tells us simply that as noted in fig. 3(a) and 3(b) the effect of the quark mass is negligible.

In order to study the restoration of the chiral symmetry and the breakdown of the chiral perturbation theory it's interesting to study the temperature dependence of the pion decay constant $f_{\pi}$ that is defined on the lattice 36] as

$$
f_{\pi}=\frac{1}{4} \frac{m_{l} \sinh \left(m_{\pi}\right)^{1 / 2}}{\sinh \left(m_{\pi} / 2\right)^{2}} \sqrt{A_{P S}}
$$

where $m_{\pi}$ and $A_{P S} 9$ are respectively the mass and the amplitude extracted from the Goldstone pion correlator. This relation is nothing else than the staggered lattice version [36] of the continuum definition through the coupling of the axial current to the pion

$$
\sqrt{2} f_{\pi} m_{\pi}=-\left\langle 0\left|\bar{u} \gamma_{4} \gamma_{5} d\right| \pi^{+}\right\rangle
$$

9 The amplitude $A_{P S}$ is related to $A_{N O(O)}$ (equation 8) trough the relation $A_{N O(O)}=2 A_{P S} e^{-m_{\pi} N_{s} / 2}$ 


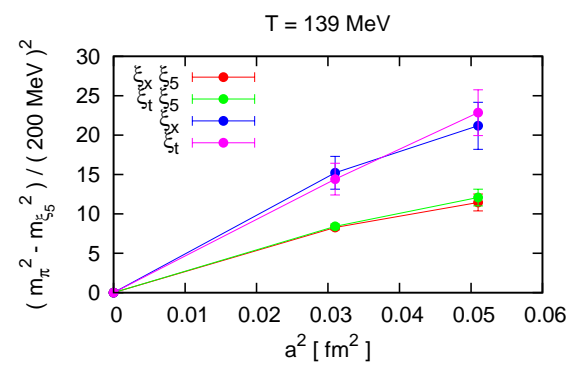

(a)

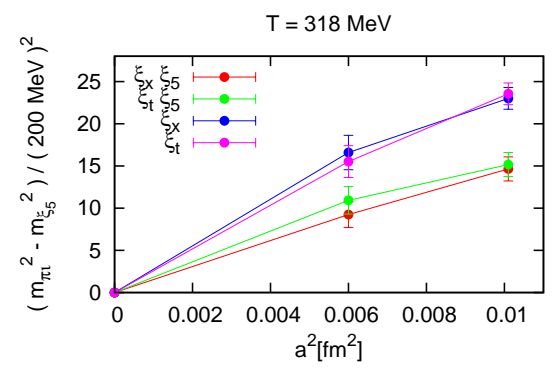

(d)

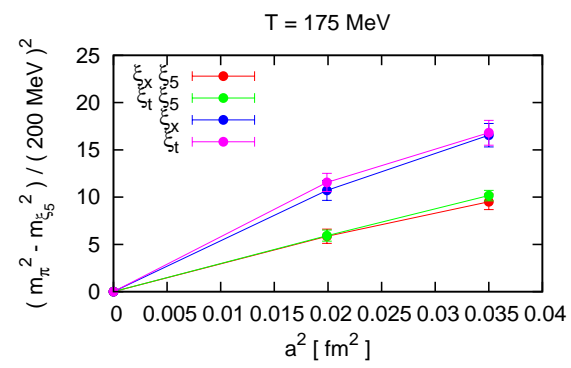

(b)

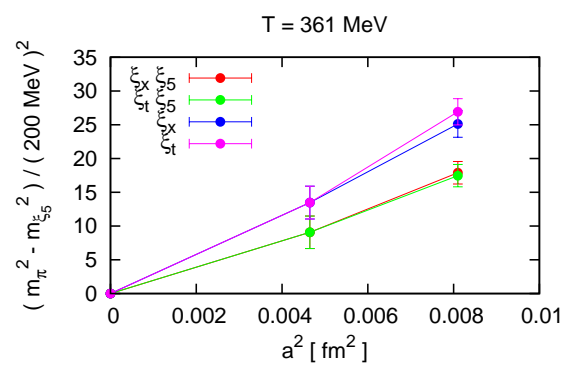

(e)

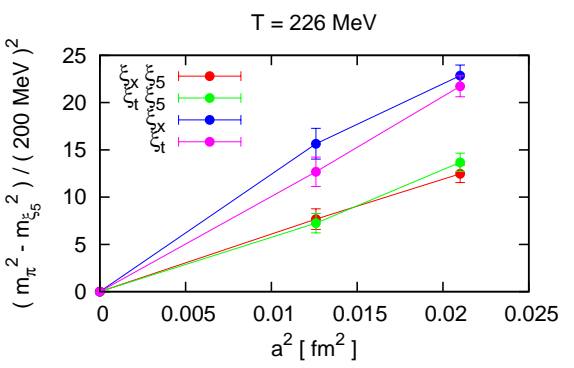

(c)

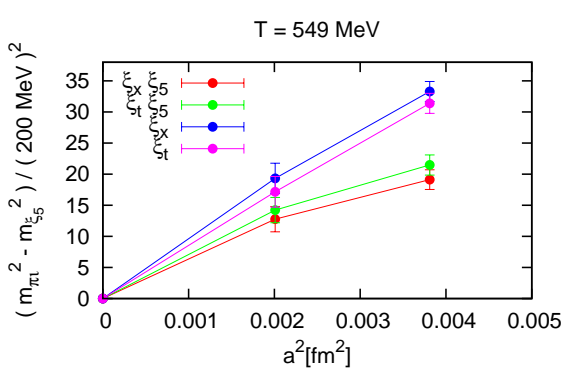

(f)

Fig. 5. Effect of the lattice spacing on the taste violation at fixed temperature values : (a) $\mathrm{T}=139 \mathrm{MeV},(\mathrm{b}) \mathrm{T}=175 \mathrm{MeV}$, (c) $\mathrm{T}=226 \mathrm{MeV}$, (d) $\mathrm{T}=361 \mathrm{MeV}$.

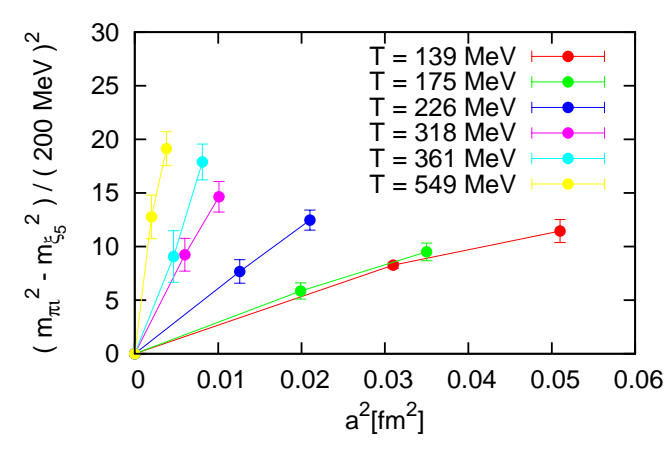

(a)

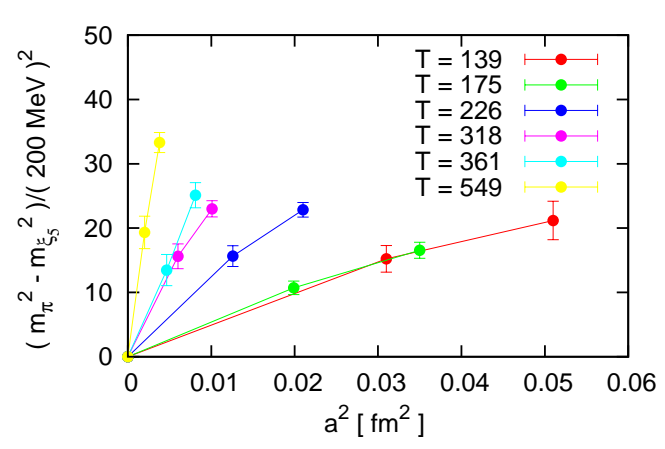

(b)

Fig. 6. Lattice spacing dependence of the taste splitting for one link $\left(m_{\gamma_{5} \otimes \xi_{x} \xi_{5}}^{2}-m_{\gamma_{5} \otimes \xi_{5}}^{2}\right)$ (e) and two link meson operators $\left(m_{\gamma_{5} \otimes \xi_{x}}^{2}-m_{\gamma_{5} \otimes \xi_{5}}^{2}\right)$ at different temperatures.

From figure 8(a) one can essentially note the sharp decrease of the value of $f_{\pi}$ in the proximity of the transition and a near vanishing after that point. This signals clearly that the chiral symmetry is restored and the chiral perturbation theory is no more applicable. In the chiral symmetry broken phase even if only few data are available we can observe a temperature dependence of $f_{\pi}(T)$.
Another thing to point out is the difference between the $N_{\tau}=8$ and the $N_{\tau}=6$ results. This is probably due to the fact that in the first case the discretization errors are reduced compared to the second lattice and indeed the values are closer to the continuum value of $f_{\pi}(0) \simeq 93$ $\mathrm{MeV}$. It would be interesting to get some more data in the confined phase and to perform an extrapolation to 


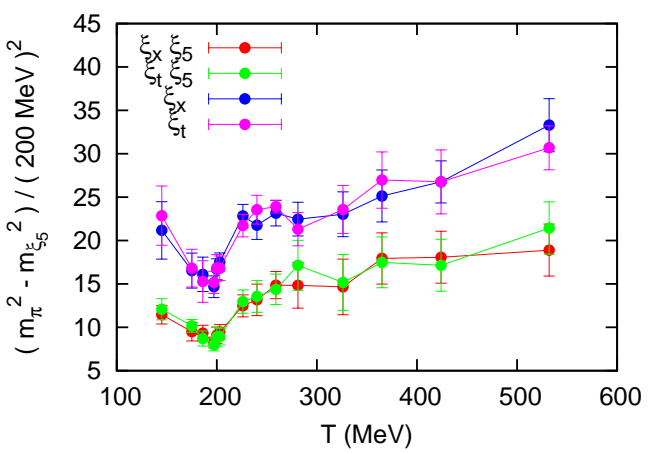

(a)

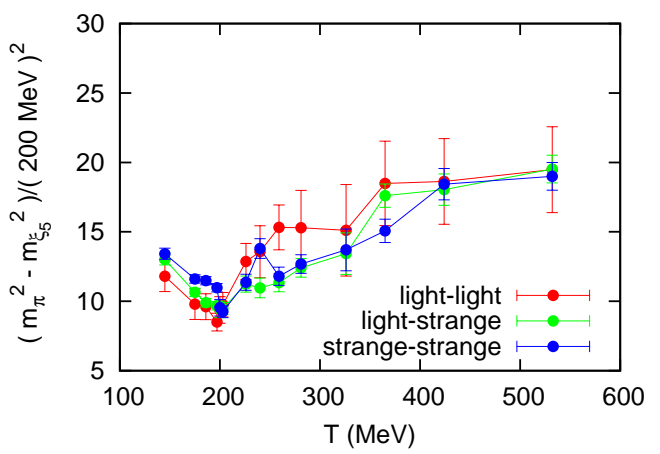

(b)

Fig. 7. (a) Effect of the temperature on the taste violation in the pion channel at $N_{\tau}=6$. (b) Flavor dependence of the taste violation for the light-light, light-strange and strange-strange pseudoscalar channels. The quantity $\Delta_{\xi_{x}}=m_{\gamma_{5} \otimes \xi_{x}}^{2}-m_{\gamma_{5} \otimes \gamma_{5}}^{2}$ is plotted for different flavor content at $N_{\tau}=6$

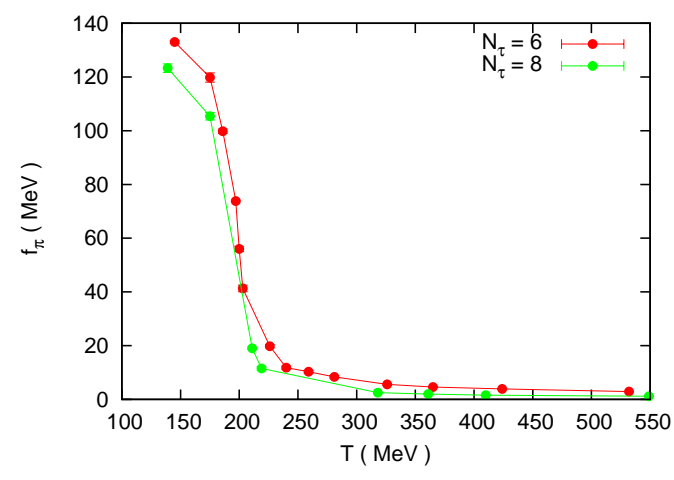

(a)

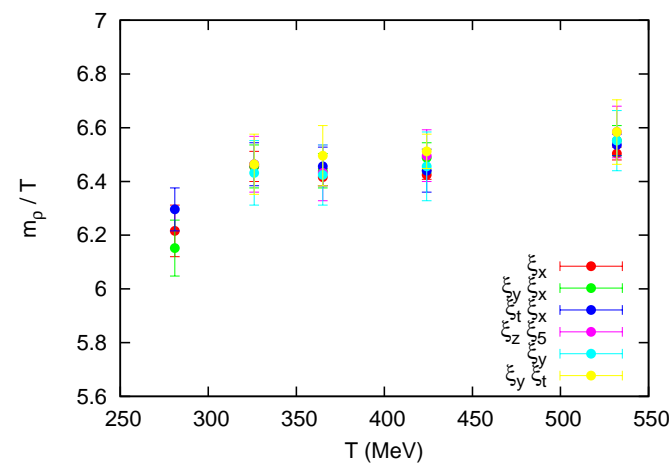

(b)

Fig. 8. (a) Temperature dependence of the pion decay constant $f_{\pi}$ in $\mathrm{MeV}$ calculated at $N_{\tau}=6$. (b) Temperature dependence for different taste state in the vector channel identified by the spin $\times$ taste matrix $\gamma_{x} \otimes[\xi]$ calculated at $N_{\tau}=6$.

the physical mass point and to the continuum similar to that done at zero temperature in 38 .

In the last picture we plot the temperature dependence of the screening masses for some taste states in the vector channel. Unfortunately since at low temperatures these correlators are very noisy it is in general difficult to extract the vector screening masses for $T<2 T_{c}$. Looking at figure 8(b) we can essentially note two facts: the first is that at temperatures between 2 and $3 T_{c}$ the values of the screening masses in the vector channel are more or less constant and have already reached a value of $2 \pi T$ which is the infinite temperature value in the thermodynamic limit. The second point is that taste violations do not seem to be present in these channels since the difference between the various tastes are within the error bars. We have also looked at other vector and axial-vector channels finding analogous results.

\section{Summary}

In this paper we have investigated the violation of taste symmetry at finite temperature in $2+1$ flavor QCD utilizing the gauge configurations generated by the RBCBielefeld 30, 33 and the HotQCD 34 collaborations using the improved $\mathrm{p} 4$ fermion action. Two different lattice sizes, $24^{3} \times 6$ and $32^{3} \times 8$, have been used to understand the role of the temperature in the taste breaking phenomena. We have calculated the screening masses by fitting the spatial meson correlators and even if we have been primarily concentrated on the pion channels, some other mesonic states built from strange quarks and some scalar, axial-vector and vector operators have been considered.

The analysis of the taste symmetry breaking is important since this violation is related to the cut-off dependence of the physical quantities. At temperatures below the transition our aim was to understand whether the predictions 
of the staggered chiral perturbation theory on the taste violation are still valid, with possibly temperature dependent effective low energy constants, or whether eventually some new terms in the chiral lagrangian would have to be considered. As pointed out in the previous section for $T<T_{c}$ we have found indications that the taste splitting in the pion channel has an $a^{2}$ behavior as predicted by $\mathrm{S} \chi \mathrm{PT}$ and no effects on the temperature can be observed in this region. It could be interesting to compare the results with zero temperature taste splittings with the same action.

The apparent temperature independence of the taste splittings in combination with a noticeably temperature dependent pion decay constant (fig. 8(a) implies within staggered chiral perturbation theory that the coefficients $C_{i}$ of the lagrangian (12) acquire a temperature dependence. However, as not all taste states in the pion multiplet are accessible at high temperature due to their dying out when the transition region is approached, the system of equations for the $C_{i}$ cannot be solved and it is not possible to determine the temperature behavior of these LECs. Above the transition the situation changes drastically. As also indicated by the vanishing of the pion decay constant $f_{\pi}$, chiral symmetry is restored and the predictions of chiral perturbation theory are no longer valid. Staying nevertheless with the chirally inspired definition of taste splittings, eq. (22), this quantity measures taste splittings that behave like $a^{2}$ - as is expected - but with a slope which increases with temperature, i.e. the splittings appear to be temperature dependent. However, because chiral symmetry is restored in this region the pion ceases to be a Goldstone particle and acquires a non-vanishing mass. For sufficiently high temperatures the screening masses will become proportional to the temperature. As a consequence, the splittings as defined in (22) should then behave according to

$$
\Delta_{[\xi]} \sim m_{\xi_{5}}\left(m_{[\xi]}-m_{\xi_{5}}\right) \sim T\left(m_{[\xi]}-m_{\xi_{5}}\right) \gtrsim a^{2} T
$$

i.e. rise with temperature. The temperature interval investigated, $T_{c}<T<2 T_{c}$, represents the transition region where the pion changes from Goldstone nature to approaching free theory behavior such that the temperature dependence is more complicated and a linear rise not yet clearly identifiable. It would be interesting to better understand the behavior in the chirally restored phase and to compare for instance with perturbative predictions which could guide the numerical analysis and should be reliable at high temperature.

Another point that we have analyzed carefully is the finite temperature splitting of the $T=0$ multiplets 29 . According to group theory e.g. the triplets of the $T=0$ GTS split into a two plus a one dimensional irrep of the finite temperature GTS. As was already noted in the literature 35 for some local axial-vector and vector channels a splitting between "longitudinal" and "transverse" screening masses has been observed. Indeed, also in the other, non-local triplet channels $(n=5,6,11,12,19,20)$ these splittings can be seen even if the analysis is performed with point source operators where only few data in the high $T$ regime are available. The situation may improve with the use of wall sources succeeding to analyze configurations at lower temperatures. Quite surprisingly, however, we found that pion triplets $n=7,8,13,14$ do not split in this way. Indeed the difference between the "transversal" and the "longitudinal" screening masses is compatible within the error bars. This is a clear indication that at finite $T$ at least in the pion channel, contrary to group theory arguments, an enhancement of the $S O(2) \times Z_{2}$ symmetry occurs and a restoration of an $S O(3)$ symmetry can be observed in these channels. As already stressed previously we don't have access to all the taste masses, so that we cannot check for a complete $S O(4)$ restoration like in the zero temperature case.

Finally we have evaluated the flavor effect on the taste splitting. For temperatures above $1.5 T_{c}$ the splittings are flavor independent for the simple reason that in this region the effect of the quark mass is negligible. For temperatures below the transition the fact that no flavor effect can be observed is non-trivial and supports the applicability of staggered chiral perturbation theory, which predicts that the pion splittings are independent of the flavor of the quark constituents, in this temperature regime.

\section{Acknowledgments}

This work is supported by the Research Executive Agency (REA) of the European Union under Grant Agreement PITNGA- 2009-238353 (ITN STRONGnet). F.P. would like to thank the hospitality of the G. Galilei Institute for Theoretical Physics, Florence. The numerical computations have been carried out on the apeNEXT at Bielefeld University.

\section{Appendix}

In the tables 3 and 4 we have summarized the pion screening masses for different taste states. The screening masses are given in lattice units and the data can be easily converted to either temperature or vacuum $\left(r_{0}\right)$ units by means of the included zero temperature results for $r_{0} / a$ from 33 .

\section{References}

1. J. B. Kogut and L. Susskind, Phys. Rev. D 11 (1975) 395.

2. B. Bunk, M. Della Morte, K. Jansen and F. Knechtli, Nucl. Phys. B 697 (2004) 343.

3. D. H. Adams, Phys. Rev. D 72 (2005) 114512.

4. F. Maresca and M. Peardon, arXiv:hep-lat/0411029.

5. Y. Shamir, Phys. Rev. D 71 (2005) 034509.

6. H. Neuberger, Phys. Rev. D 70 (2004) 097504.

7. C. Bernard et al., PoS LAT2005 (2006) 114.

8. C. Bernard, Phys. Rev. D 71 (2005) 094020.

9. S. Durr and C. Hoelbling, Phys. Rev. D 71 (2005) 054501.

10. M. Creutz, Phys. Lett. B 649 (2007) 230.

11. M. Creutz, PoS LAT2007 (2007) 007. 


\begin{tabular}{|lc|cccccc|}
\hline$\beta$ & $r_{0} / a$ & $\begin{array}{c}a M_{P S} \\
{\left[\gamma_{5} \otimes \xi_{5}\right]}\end{array}$ & $\begin{array}{c}a M_{\pi} \\
{\left[\gamma_{5} \otimes \xi_{x} \xi_{5}\right]}\end{array}$ & $\begin{array}{c}a M_{\pi} \\
{\left[\gamma_{5} \otimes \xi_{t} \xi_{5}\right]}\end{array}$ & $\begin{array}{c}a M_{\pi} \\
{\left[\gamma_{5} \otimes \xi_{x}\right]}\end{array}$ & $\begin{array}{c}a M_{\pi} \\
{\left[\gamma_{5} \otimes \xi_{t}\right]}\end{array}$ & $\begin{array}{c}a M_{\pi} \\
{\left[\gamma_{5} \otimes 1\right]}\end{array}$ \\
\hline \hline \multicolumn{7}{|c}{$32^{3} \times 8$} \\
\hline 3.430 & 2.647 & $0.201(01)$ & $0.555(12)$ & $0.559(10)$ & $0.730(60)$ & $0.712(52)$ & - \\
3.500 & 3.328 & $0.175(01)$ & $0.382(12)$ & $0.390(12)$ & $0.517(40)$ & $0.500(40)$ & - \\
3.530 & 3.654 & - & $0.354(10)$ & $0.370(12)$ & $0.461(30)$ & $0.454(28)$ & - \\
3.570 & 4.009 & $0.311(14)$ & $0.455(09)$ & $0.445(08)$ & $0.517(18)$ & $0.503(22)$ & $0.587(30)$ \\
3.585 & 4.160 & $0.351(10)$ & $0.473(14)$ & $0.467(13)$ & $0.573(21)$ & $0.538(23)$ & $0.606(30)$ \\
3.760 & 6.050 & $0.614(08)$ & $0.659(07)$ & $0.670(08)$ & $0.697(10)$ & $0.688(10)$ & $0.709(07)$ \\
3.820 & 6.864 & $0.666(06)$ & $0.698(07)$ & $0.698(06)$ & $0.713(07)$ & $0.713(06)$ & $0.723(06)$ \\
3.920 & 7.814 & $0.702(03)$ & $0.722(06)$ & $0.724(06)$ & $0.735(05)$ & $0.733(04)$ & $0.745(05)$ \\
4.000 & 9.048 & $0.721(02)$ & $0.744(03)$ & $0.746(02)$ & $0.756(03)$ & $0.754(03)$ & $0.761(03)$ \\
4.080 & 10.390 & $0.740(03)$ & $0.758(03)$ & $0.759(03)$ & $0.767(03)$ & $0.764(02)$ & $0.772(03)$ \\
\hline
\end{tabular}

Table 3. Screening masses for some taste states in the pion multiplet from $N_{\tau}=8$ lattices.

\begin{tabular}{|lc|ccccc|}
\hline$\beta$ & $r_{0} / a$ & $\begin{array}{c}a M_{P S} \\
{\left[\gamma_{5} \otimes \xi_{5}\right]}\end{array}$ & $\begin{array}{c}a M_{\pi} \\
{\left[\gamma_{5} \otimes \xi_{x} \xi_{5}\right]}\end{array}$ & $\begin{array}{c}a M_{\pi} \\
{\left[\gamma_{5} \otimes \xi_{t} \xi_{5}\right]}\end{array}$ & $\begin{array}{c}a M_{\pi} \\
{\left[\gamma_{5} \otimes \xi_{x}\right]}\end{array}$ & $\begin{array}{c}a M_{\pi} \\
{\left[\gamma_{5} \otimes \xi_{t}\right]}\end{array}$ \\
\hline \hline \multicolumn{7}{|c|}{$24^{3} \times 6$} \\
\hline 3.351 & 2.069 & $0.257(01)$ & $0.820(34)$ & $0.840(38)$ & $1.090(80)$ & $1.130(80)$ \\
3.410 & 2.503 & $0.222(03)$ & $0.627(30)$ & $0.646(20)$ & $0.805(45)$ & $0.811(48)$ \\
3.430 & 2.647 & $0.221(06)$ & $0.592(24)$ & $0.575(24)$ & $0.755(42)$ & $0.737(52)$ \\
3.445 & 2.813 & $0.255(07)$ & $0.549(14)$ & $0.542(16)$ & $0.697(24)$ & $0.707(24)$ \\
3.455 & 2.856 & $0.281(07)$ & $0.576(20)$ & $0.571(24)$ & $0.737(20)$ & $0.740(26)$ \\
3.460 & 2.890 & $0.321(10)$ & $0.600(15)$ & $0.588(18)$ & $0.761(15)$ & $0.748(22)$ \\
3.490 & 3.223 & $0.436(08)$ & $0.680(14)$ & $0.688(15)$ & $0.830(12)$ & $0.815(10)$ \\
3.510 & 3.426 & $0.551(10)$ & $0.747(16)$ & $0.752(16)$ & $0.851(12)$ & $0.871(12)$ \\
3.540 & 3.687 & $0.630(08)$ & $0.803(10)$ & $0.798(12)$ & $0.885(08)$ & $0.892(12)$ \\
3.570 & 4.009 & $0.720(08)$ & $0.853(13)$ & $0.872(15)$ & $0.914(10)$ & $0.905(07)$ \\
3.630 & 4.651 & $0.799(10)$ & $0.890(08)$ & $0.893(09)$ & $0.938(07)$ & $0.941(07)$ \\
3.690 & 5.201 & $0.842(07)$ & $0.927(06)$ & $0.925(05)$ & $0.959(08)$ & $0.967(07)$ \\
3.760 & 6.050 & $0.887(04)$ & $0.948(03)$ & $0.945(03)$ & $0.976(04)$ & $0.976(03)$ \\
3.920 & 7.590 & $0.960(02)$ & $0.998(03)$ & $1.003(04)$ & $1.026(04)$ & $1.021(04)$ \\
\hline
\end{tabular}

Table 4. Screening masses for some taste states in the pion multiplet from $N_{\tau}=6$ lattices.

12. C. Bernard, M. Golterman, Y. Shamir and S. R. Sharpe, Phys. Lett. B 649 (2007) 235.

13. M. Creutz, Phys. Lett. B 649 (2007) 241.

14. M. F. L. Golterman, Nucl. Phys. B 273 (1986) 663.

15. C. Aubin et al. [MILC Collaboration], Phys. Rev. D 70 (2004) 114501.

16. A. Bazavov and P. Petreczky [for the HotQCD Collaboration], PoS LATTICE2010, 169 (2010).

17. A. Bazavov et al. [MILC Collaboration], Phys. Rev. D 82 (2010) 074501 arXiv:1004.0342 [hep-lat]].

18. U. M. Heller, F. Karsch and B. Sturm, Phys. Rev. D 60 (1999) 114502.

19. F. Karsch, E. Laermann and A. Peikert, Phys. Lett. B 478 (2000) 447.

20. K. Orginos, D. Toussaint and R. L. Sugar [MILC Collaboration], Phys. Rev. D 60 (1999) 054503.

21. Y. Aoki, Z. Fodor, S. D. Katz and K. K. Szabo, JHEP 0601 (2006) 089.

22. E. Follana et al. [HPQCD Collaboration and UKQCD Collaboration], Phys. Rev. D 75 (2007) 054502.

23. W. J. Lee and S. R. Sharpe, Phys. Rev. D 60 (1999) 114503.
24. C. Aubin and C. Bernard, Phys. Rev. D 68 (2003) 034014. 25. C. Bernard, C. E. DeTar, Z. Fu and S. Prelovsek, Phys. Rev. D 76 (2007) 094504 arXiv:0707.2402 [hep-lat]].

26. C. Bernard [MILC Collaboration], Phys. Rev. D 65 (2002) 054031 .

27. C. W. Bernard et al., Phys. Rev. D 64, 054506 (2001).

28. M. F. L. Golterman and J. Smit, Nucl. Phys. B 255 (1985) 328.

29. S. Gupta, Phys. Rev. D 60, 094505 (1999).

30. M. Cheng et al., Phys. Rev. D78 (2008) 034506.

31. R. Altmeyer et al., Nucl. Phys. B389 (1993) 445.

32. I. Horváth, A. D. Kennedy and S. Sint, Nucl. Phys. Proc. Suppl. 73 (1999) 834; M. A. Clark, A. D. Kennedy and Z. Sroczynski, Nucl. Phys. Proc. Suppl. 140 (2005) 835; M. A. Clark and A. D. Kennedy, Phys. Rev. Lett. 98 (2007) 051601.

33. M. Cheng et al., Phys. Rev. D77 (2008) 014511.

34. A. Bazavov et al., Phys. Rev. D80 (2009) 014504.

35. M. Cheng et al., Eur. Phys. J. C 71 (2011) 1564.

36. G. W. Kilcup and S. R. Sharpe, Nucl. Phys. B 283 (1987) 493.

37. C. Amsler et al. [Particle Data Group], Phys. Lett. B 667 (2008) 1. 
38. A. Bazavov, D. Toussaint, C. Bernard, J. Laiho, C. DeTar, L. Levkova, M. B. Oktay and S. Gottlieb et al., Rev. Mod. Phys. 82 (2010) 1349 